Génét. Sél. Evol., 1983, 15 (1), 99-118

\title{
Dix ans d'une expérience de sélection individuelle sur des verrats utilisés en insémination artificielle. \\ II. Paramètres génétiques estimés
}

\author{
L. OLLIVIER \\ avec la collaboration technique de Marie-Reine Perretant, J. Gruand et C. Felgines \\ I.N.R.A., Station de Génétique quantitative et appliquée, \\ Centre de Recherches zootechniques, F 78350 Jouy-en-Josas
}

\begin{abstract}
Résumé
Les résultats recueillis entre 1966 et 1975 dans une expérience de sélection réalisée au centre d'insémination artificielle porcine I.N.R.A. de Rouilié (Vienne) sont analysés en vue d'estimer les héritabilités $\left(\mathrm{h}^{2}\right)$ et corrélations génétiques $\left(\mathrm{r}_{\mathrm{A}}\right)$ relatives à des variables $\mathrm{X}$ mesurées sur les verrats - vitesse de croissance de 30 à $80 \mathrm{~kg}\left(\mathrm{X}_{1}\right)$ et épaisseur du gras dorsal à $80 \mathrm{~kg}\left(\mathrm{X}_{3}\right)$ - et à un ensemble de 22 variables $\mathrm{Y}$ mesurées sur des mâles castrés et des femelles abattus au poids vif de $100 \mathrm{~kg}$. Les effectifs totaux sont de 850 pour $X$ et 2732 pour $\mathrm{Y}$, tous ces individus étant issus de 94 pères (dont 85 eux-mêmes mesurés pour $\mathrm{X}$ ). Le dispositif permet de calculer des covariances entre parent et descendant d'une part et entre demi-germains d'autre part. Ces covariances sont d'abord corrigées pour les effets de la sélection des verrats puis combinées entre elles pour obtenir, par les méthodes classiques, $h^{2}$ ou $r_{A}$. Cependant, pour les variances génétiques de $X$, la covariance génétique entre $X_{1}$ et $X_{3}$ et les covariances génétiques entre $\mathrm{X}$ et $\mathrm{Y}$, il existe plusieurs estimateurs du même paramètre. Ceux-ci sont combinés entre eux par la méthode des moindres carrés généralisés, selon une procédure itérative. Les $r_{A}$ entre les variables $X$ et $Y$ tendent à indiquer l'absence d'interaction génotype $\times$ sexe ou génotype $\times$ période de contrôle pour les caractères de croissance et d'adiposité. Les $r_{A}$ entre $X_{1}$ et $X_{3}$ d'une part et les mesures de qualité de la viande d'autre part ( $Y_{15}$ à $\left.Y_{22}\right)$ sont, dans l'ensemble, défavorables : pour $\mathrm{X}_{1}$ les corrélations les plus élevées sont obtenues avec le $\mathrm{pH}(-0,22$ pour l'adductor femoris $)$ et la réflectance $(0,22$ pour le biceps femoris $)$, pour $\mathrm{X}_{3}$ avec le temps d'imbibition $(0,52$ pour le gluteus superficialis).
\end{abstract}

Mots-clés : Héritabilité, corrélation génétique, croissance, carcasse, porc.

\section{Summary}

A ten-year experiment on individual selection

of boars used in artificial insemination

\section{Estimated genetic parameters}

Heritabilities $\left(h^{2}\right)$ and genetic correlations $\left(r_{\Lambda}\right)$ are derived from the results of a selection experiment carried on at a pig artificial insemination center belonging to I.N.R.A. (located at Rouillé, Vienne, France). The data, covering the period 1966-1975, include 850 boars 
measured for a set of $\mathrm{X}$ variables - namely average daily gain from 30 to $80 \mathrm{~kg}$ liveweight $\left(X_{1}\right)$ and backfat thickness at $80 \mathrm{~kg}$ liveweight $\left(\mathrm{X}_{3}\right)-$ and 2732 female and castrated males measured, at $100 \mathrm{~kg}$ liveweight, for a set of $22 \mathrm{Y}$ variables including growth, carcass and meat quality traits. All boars, females and castrates are from 94 sires, of which 85 were themselves measured for $\mathrm{X}$. The design allows the calculation of various parent-offspring and half-sib covariances which are first corrected for the bias due to the boar selection and then combined in order to yield $\mathrm{h}^{2}$ and $\mathrm{r}_{\mathrm{A}}$ in the usual manner. However, several estimates are available for the genetic variances of $X$, the genetic covariance between $X_{1}$ and $X_{3}$, and the genetic covariances between $X$ and $Y$. These estimates are pooled by the method of generalized least-squares, iteratively, and approximate variances of the various estimators of $h^{2}$ and $r_{A}$ are derived. The $r_{A}$ 's between $X$ and $Y$ suggest a lack of genotype $X$ sex or genotype $\times$ test period interaction for growth and fatness traits. The $r_{\mathbf{A}}$ 's between $X$ and meat quality traits $\left(Y_{15}\right.$ to $\left.Y_{22}\right)$ are generally unfavourable : with $X_{1}$ the highest values are obtained for $\mathrm{pH}(-0.22$ for adductor femoris) and reflectance $(0.22$ for biceps femoris) and with $\mathbf{X}_{3}$ the highest values concern water-holding capacity $(0.52$ for gluteus superficialis).

Key-words: : Heritability, genetic correlation, growth, carcass, pig.

\section{Introduction}

Les résultats d'une expérience de sélection réalisée dans le cadre du centre d'insémination artificielle porcine de l'I.N.R.A. à Rouillé (Vienne) ont été présentés dans une étude précédente (Ollivier, 1977) retraçant l'évolution observée au cours de dix années pour différents caractères relatifs à la croissance, la carcasse et la qualité de la viande. Une interprétation génétique détaillée de ces résultats reste à faire afin de préciser les paramètres génétiques de la population porcine concernée et d'établir dans quelle mesure les réponses obtenues sont en accord avec les paramètres estimés, suivant la démarche généralement suivie dans l'analyse de toute expérience de sélection. Celle qui nous occupe est essentiellement une sélection sur un indice à deux caractères. Les réponses sont mesurées sur un grand nombre d'autres caractères, différents de ceux de l'indice de sélection. L'estimation des paramètres génétiques, héritabilités et corrélations génétiques, pour l'ensemble de ces caractères, fait l'objet du présent article. Les paramètres « realisés », qu'on peut déduire des réponses, feront l'objet du troisième volet de l'analyse.

\section{Matériel et méthodes}

Pour la description du schéma expérimental et des variables considérées nous renvoyons au précédent article de cette série (Ollivier, 1977). Parmi les variables qui y sont décrites, nous retenons pour cette analyse deux des variables "verrats ", $X_{1}$ (gain moyen quotidien de 30 à $80 \mathrm{~kg}$ ) et $X_{3}$ (épaisseur du gras dorsal à $80 \mathrm{~kg}$ ) incluses dans l'indice de sélection utilisé depuis 1969, et l'ensemble des variables « descendants " $\mathrm{Y}_{1}$ à $\mathrm{Y}_{22}$.

\section{A. Estimation des covariances entre apparentés}

Les paramètres génétiques sont déduits des covariances entre apparentés obtenues pour les deux relations parent-descendant et demi-germains. Les variables $\mathrm{X}$ étant mesurées sur les pères et sur les fils et les variables $\mathrm{Y}$ seulement sur les descendants, 
on peut définir 3 ensembles de variables aléatoires représentés par 3 vecteurs $\mathbf{X}$, $\mathbf{X}^{\prime}$ et $\mathbf{Y}$. $\mathbf{X}$ est le vecteur des variables $X_{1}$ et $X_{3}$ mesurées sur les pères. $\mathbf{X}^{\prime}$ est le vecteur des deux mêmes variables mesurées sur un fils tiré au hasard dans la descendance d'un père donné et de mères différentes. $Y$ est un vecteur de 22 variables (correspondant aux mesures $\mathrm{Y}_{1} \ldots \mathrm{Y}_{22}$ ) définies selon le même principe que pour $\mathbf{X}^{\prime}$. Les covariances entre ces 26 variables aléatoires se disposent selon le tableau ci-dessous (matrice symétrique) :

$$
\begin{array}{ccc}
\mathbf{V}_{\mathrm{xx}} & \mathbf{D}_{\mathrm{xx}} \mathbf{D}_{\mathrm{xy}} \\
- & \mathbf{G}_{\mathrm{x}^{\prime} \mathbf{x}^{\prime}} & \mathbf{G}_{\mathrm{x}^{\prime} \mathrm{y}} \\
- & - & \mathbf{G}_{\mathrm{yy}}
\end{array}
$$

dans lequel :

- $\mathbf{V}_{\mathbf{x x}}$ est la matrice $(2 \times 2)$ des variances-covariances phénotypiques de $\mathbf{X}, \mathbf{G}_{\mathbf{x}^{\prime} \mathbf{x}^{\prime}}$ et $\mathbf{G}_{\mathrm{yy}}$ sont les matrices des variances-covariances entre demi-germains pour $\mathbf{X}^{\prime}$ (de dimension $2 \times 2$ ) et pour $\mathbf{Y}($ de dimension $22 \times 22$ ) respectivement,

- $\mathbf{D}_{\mathbf{x x}}$ et $\mathbf{D}_{\mathrm{xy}}$ sont les matrices de covariance parent $(\mathbf{X})$ - descendant $\left(\mathbf{X}^{\prime}\right.$ ou $\left.\mathbf{Y}\right)$, $\mathbf{G}_{\mathbf{x}^{\prime} \mathbf{y}}$ est la matrice de covariances entre demi-germains, $\mathbf{X}^{\prime}$ étant mesuré sur l'un et $\mathbf{Y}$ sur l'autre.

Les matrices $\mathbf{G}_{x^{\prime} x^{\prime}}$ et $\mathbf{G}_{y y}$ sont les composantes paternelles de variance et de covariance obtenues en appliquant un modèle mixte de moindres carrés. Ce modèle comprend des effets fixés (l'année et une covariable poids final pour $\mathbf{X}^{\prime}$, le sexe et le facteur année-bâtiment pour $\mathbf{Y}$ ) et trois effets aléatoires emboités, soient père (intra-année ou année-bâtiment) mère et résiduel (HARVEY, 1972). La composante paternelle ainsi estimée représente donc l'effet «père » intra-année et génération (pour $\mathbf{X}^{\prime}$ ) ou intra-année-bâtiment (pour $\mathbf{Y}$ ). Précisons cependant que pour les variables "qualité de viande" $\left(\mathrm{Y}_{15}\right.$ à $\left.\mathrm{Y}_{22}\right)$ les composantes paternelles sont estimées sur les données préalablement corrigées pour les effets du sexe et de la date d'abattage estimés précédemment (Ollivier, 1977).

Pour établir les matrices $\mathbf{D}_{x^{\prime}}$ et $\mathbf{D}_{\mathrm{xy}}$ les variables $\mathbf{X}, \mathbf{X}^{\prime}$ et $\mathbf{Y}$ sont d'abord corrigées pour les effets fixés précédemment indiqués et les covariances parent-descendant sont obtenues en calculant sur les données ainsi corrigées la covariance entre le père et la moyenne de ses descendants (sans correction pour le nombre de degrés de liberté). Les covariances $\mathbf{G}_{x^{\prime} y}$ sont obtenues selon le même principe en calculant la covariance entre la moyenne des descendances paternelles pour $\mathbf{X}^{\prime}$ et pour $\mathbf{Y}$, les germains des verrats mesurés pour $\mathbf{X}^{\prime}$ étant exclus de la moyenne de descendance pour $\mathbf{Y}$. Les dix verrats fondateurs n'ayant pas été mesurés, les covariances $\mathbf{D}_{\mathrm{xx}^{\prime}}$ et $\mathbf{D}_{\mathrm{xy}}$ sont calculées sur des sous-échantillons (voir tableau 1).

\section{B. Incidence de la sélection des pères sur les covariances entre apparentés}

Les pères retenus à chaque génération étant sélectionnés (sur $X_{1}$ et $X_{3}$ ), les covariances estimées s'écartent des valeurs qui seraient obtenues si les pères étaient choisis au hasard. La correction pour cet effet de la sélection est faite, connaissant les variances-covariances des pères sélectionnés, selon une méthode générale décrite par AITKEn (1934) et dont le détail, avec une application à une sélection sur un indice à 3 caractères, est donné par ailleurs (Ollivier \& Derrien, 1981). Si, pour simplifier les notations, on désigne par $\mathbf{V}$ la matrice de variance-covariance de $\mathbf{X}$ avant sélection, par $\mathbf{S}$ la matrice équivalente pour les pères sélectionnés et par $\mathbf{D}$ et $\mathbf{G}$ respectivement 
les matrices de covariance parent-descendant et entre demi-germains (dans l'échantillon sélectionné) estimées précédemment, les covariances $\hat{\mathbf{D}}$ et $\hat{\mathbf{G}}$ correspondant à un choix aléatoire des pères sont estimées comme suit :

$$
\hat{\mathbf{D}}=\mathbf{V ~ S ^ { - 1 }} \mathbf{D}
$$

et :

$$
\hat{\mathbf{G}}=\mathbf{G}+\hat{\mathbf{D}}^{\prime}\left(\mathbf{V}^{-1}-\mathbf{V}^{-1} \mathbf{S} \mathbf{V}^{-1}\right) \hat{\mathbf{D}}
$$

\section{TABLeau 1}

Echantillons utilisés pour estimer les covariances entre apparentés.

\begin{tabular}{|c|c|c|c|c|c|}
\hline $\begin{array}{l}\text { Génération } \\
\text { Generation }\end{array}$ & $\begin{array}{l}\text { Variable } \\
\text { Variable }\end{array}$ & \multicolumn{4}{|c|}{$\begin{array}{l}\text { Echantillon utilisé pour estimer } \\
\text { Sample used to estimate }\end{array}$} \\
\hline \multirow{6}{*}{$\begin{array}{l}\text { Père (Sire) } \ldots \ldots \ldots \ldots \\
\text { Descendance (Progeny) }\end{array}$} & & $\mathbf{G}_{x^{\prime} x^{\prime}}$ et $\mathbf{G}_{y y}$ & $\mathbf{D}_{\mathrm{xx}^{\prime}}$ & $\mathbf{D}_{\mathrm{xy}}$ & $\mathbf{G}_{x^{\prime} y}$ \\
\hline & $\mathrm{X}$ & $94^{(1)}$ & 85 & $84^{(2)}$ & $88^{(3)}$ \\
\hline & $\mathrm{X}^{\prime}$ & 850 & 798 & - & 795 \\
\hline & $Y_{1} \ldots Y_{14}$ & 2732 & - & 2372 & 1665 \\
\hline & $Y_{15} \ldots Y_{20}$ & 1929 & - & 1942 & 907 \\
\hline & $Y_{21} \ldots Y_{22}$ & 1691 & - & 1703 & 907 \\
\hline
\end{tabular}

Samples used to estimate the covariances between relatives.

(1) 95 pour $\mathrm{X}^{\prime}, 72$ pour $\mathrm{Y}_{15} \ldots \mathrm{Y}_{22}$

95 for $\mathrm{X}^{\prime}, 72$ for $\mathrm{Y}_{15} \ldots \mathrm{Y}_{2 ?}$ ?

(2) 72 pour $\mathrm{Y}_{15} \ldots \mathrm{Y}_{22}$

72 for $Y_{15} \ldots Y_{22}$

(3) 62 pour $Y_{15} \ldots Y_{22}$

62 for $Y_{15} \ldots Y_{22}$

Les symboles des covariances sont donnés dans le texte.

Symbols for covariances in the text.

\section{Estimation des paramètres génétiques \\ (héritabilités et corrélations génétiques)}

Pour les variables $\mathbf{Y}$ une seule estimation des variances et covariances génétiques est disponible, qui est donnée par $4 \hat{\mathbf{G}}_{\mathrm{yy}}$.

Pour les variables $\mathbf{X}$ on dispose de deux estimations des variances génétiques (les termes diagonaux de $2 \hat{\mathbf{D}}_{\mathrm{xx}^{\prime}}$ et $4 \hat{\mathbf{G}}_{\mathrm{x}^{\prime} \mathrm{x}^{\prime}}$ ) et de trois estimations de covariances génétiques (une covariance entre demi-germains et deux covariances parent-descendant, la matrice $\hat{\mathbf{D}}_{\mathrm{xx}^{\prime}}$ n'étant pas symétrique).

Pour les covariances génétiques entre $\mathbf{X}$ et $\mathbf{Y}$ on dispose de deux estimations, $4 \hat{\mathbf{G}}_{x^{\prime} y}$ et $2 \hat{\mathbf{D}}_{\mathrm{xy}}$. 
Les estimations multiples ainsi obtenues pour un même paramètre peuvent être combinées en une valeur unique par différentes méthodes. C'est la méthode des moindres carrés généralisés qui est retenue ici, selon la procédure générale décrite par Gianola (1979). Le calcul requiert la connaissance des variances et covariances des différents estimateurs, éléments qui sont donnés dans l'annexe I. Ces variances et covariances n'étant pas connues, il est nécessaire de procéder par itération. Les équations (I 3), (I 4) et (I 5) de l'annexe I sont d'abord calculées à partir des estimations D et $\mathbf{G}$ présentées plus haut dans (1), car les paramètres entrant dans ces équations doivent être les variances et covariances relatives à l'échantillon sélectionné. Une première estimation est ainsi obtenue, qui combine les estimées $\hat{\mathbf{D}}$ et $\hat{\mathbf{G}}$ de (2). $\mathbf{A}$ l'étape suivante, les paramètres à introduire dans (I 3), (I 4) et (I 5) sont déduits de cette première estimation (qui représente la vraie valeur dans la population non sélectionnée), en appliquant les corrections appropriées qui résultent de la sélection effectuée sur les pères (selon la méthode classique décrite par Ollivier \& Derrien, 1981). On procède de même avec la nouvelle estimation obtenue jusqu'à ce que le paramètre estimé se stabilise.

\section{TABLEAU 2}

Variances et covariances des variables "verrats".

Variances and covariances of the boar variables.

\begin{tabular}{|c|c|c|c|c|c|}
\hline & & \multicolumn{2}{|c|}{ Père $(\mathrm{P})$ (Sire) } & \multicolumn{2}{|c|}{ Descendant (D) (Offspring) } \\
\hline & & $x_{1}$ & $\mathrm{X}_{3}$ & $\mathrm{X}^{\prime}{ }_{1}$ & $\mathrm{X}_{3}^{\prime}$ \\
\hline \multirow{2}{*}{$\mathbf{P}$} & $X_{1}$ & 0,008075 & 0,11046 & 0,001175 & 0,03589 \\
\hline & $X_{3}$ & $(*)$ & $\begin{array}{l}5,9174 \\
8,1405\end{array}$ & $\begin{array}{l}0,03854 \\
0,05029\end{array}$ & $\begin{array}{l}1,909 \\
2,625\end{array}$ \\
\hline \multirow{2}{*}{$\mathrm{D}$} & $X^{\prime}$ & $(*)$ & $(*)$ & $\begin{array}{l}0,000162 \\
0,000241\end{array}$ & $\begin{array}{l}0,01506 \\
0,01885\end{array}$ \\
\hline & $X_{3}^{\prime}$ & $\left({ }^{*}\right)$ & $\left({ }^{*}\right)$ & $\left({ }^{*}\right)$ & $\begin{array}{l}1,064 \\
1,295\end{array}$ \\
\hline
\end{tabular}

$\mathrm{I}^{\text {re }}$ ligne : Variances-covariances estimées sur les pères sélectionnés (matrice $\mathbf{S}$ pour les variables $\mathbf{X}$ ) et leurs descendants (matrices $\mathbf{D}_{\mathbf{x} \mathbf{x}^{\prime}}$ et $\mathbf{G}_{\mathbf{x}^{\prime} \mathbf{x}^{\prime}}$ ).

Ist line : Covariances estimated on selected sires and their progeny.

$2^{\mathrm{e}}$ ligne : Variances-covariances calculées sur l'ensemble des pères contrôlés (matrice $\mathbf{V}$ pour $\mathbf{X}$ ) et corrigées pour les effets de la sélection pour les lignes et colonnes $\mathrm{X}^{\prime}{ }_{1}$ et $\mathrm{X}_{3}^{\prime}$ (matrices $\hat{\mathbf{D}}_{\mathrm{xx}^{\prime}}$ et $\hat{\mathbf{G}}_{\mathrm{x}^{\prime} \mathrm{x}^{\prime}}$ ).

2nd line: Covariances estimated on the whole sample of tested boars (for $\boldsymbol{X}$ ) and corrected for selection bias in lines (and columns) $X_{1}^{\prime}$ and $X_{3}^{\prime}$.

$X_{1}$ et $X_{1}^{\prime}$ : Gain moyen quotidien $30-80 \mathrm{~kg}(\mathrm{~kg})$.

Average daily gain $30-80 \mathrm{~kg}$.

$\mathrm{X}_{3}$ et $\mathrm{X}_{3}^{\prime}$ : Epdisseur de gras à $80 \mathrm{~kg}(\mathrm{~mm})$. Backfat thickness at $80 \mathrm{~kg}$.

(*) Valeur obtenue en permutant la ligne et la colonne.

Value obtained by permutation of line and column. 
A partir des variances et covariances génétiques ainsi estimées, les héritabilités et les corrélations génétiques sont calculées et leurs variances d'échantillonnage sont obtenues en faisant les approximations à des combinaisons linéaires des paramètres précédemment estimés, comme indiqué dans les annexes II et III.

\section{Résultats}

\section{A. Variables «verrats" $\left(X_{1}\right.$ et $\left.X_{3}\right)$}

Le tableau 2 donne les estimées obtenues pour les matrices $\mathbf{D}_{\mathrm{xx}^{\prime}}$ et $\mathbf{G}_{\mathbf{x}^{\prime} \mathbf{x}^{\prime}}$ définies en (1) ainsi que les estimées correspondantes - $\hat{\mathbf{D}}$ et $\hat{\mathbf{G}}$ définies en (2) - corrigées pour les effets de la sélection des pères. Les paramètres génétiques, déduits de ces estimées selon les méthodes détaillées dans les annexes I, II et III, sont donnés dans le tableau 3. On note pour $X_{1}$ que l'héritabilité basée sur la covariance entre demi-germains $(0,09)$ est très inférieure à celle déduite de la covariance parent-descendant $(0,26)$.

\section{TABLeau 3}

Paramètres génétiques des variables "verrats"(estimées combinées).

Genetic parameters of the boar variables (combined estimates).

\begin{tabular}{|c|c|c|c|}
\hline \multirow{3}{*}{$X_{1}(\mathrm{~kg})$} & $x_{1}$ & \multicolumn{2}{|c|}{$\mathrm{X}_{3}$} \\
\hline & $0,002468 \pm 0,0087$ & 0,07 & $\pm 0,0213$ \\
\hline & $0,21 \pm 0,07$ & 0,68 & $\pm 0,10$ \\
\hline $\mathrm{X}_{3}(\mathrm{~mm})$ & $(*)$ & $\begin{array}{l}5,24 \\
0,61\end{array}$ & $\begin{array}{l} \pm 0,87 \\
\pm 0,09\end{array}$ \\
\hline
\end{tabular}

Variables définies au tableau 2.

Variables defined in table 2.

$\mathrm{I}^{\text {re }}$ ligne : Variance-covariance génétique ( \pm erreur standard).

Ist line : Genetic variance-covariance ( \pm s.e.).

$2^{\mathrm{e}}$ ligne : Héritabilité et corrélation génétique ( \pm erreur standard).

2nd line: Heritability and genetic correlation ( \pm s.e.).

\section{B. Variables "descendants"}

Le tableau 4 donne les héritabilités des 22 variables "descendants », directement déduites des estimées $\hat{\mathbf{G}}_{\mathrm{yy}}$ corrigées pour les effets de la sélection. La comparaison des résultats concernant les vitesses de croissance, $X_{1}$ au tableau 3 et $Y_{1}$ au tableau 4 , illustre le gain de précision qu'apporte l'estimation combinée d'héritabilité. Les héritabilités de $X_{1}$ et de $Y_{1}$ sont en effet estimées avec la même précision alors que les tailles d'échantillon sont dans le rapport 1 pour $X_{1}: 3$ pour $Y_{1}$. 
Les corrélations phénotypiques et génétiques entre ces variables sont données dans les tableaux IV 1, IV 2 et IV 3 de l'annexe IV, à l'exception des corrélations phénotypiques entre les variables $Y_{1}$ à $Y_{14}$ et les variables "qualité de viande", qui sont toutes très faibles. Il faut noter que l'âge au début du contrôle est une variable qui n'a pas été considérée dans cette analyse. Toutes les corrélations phénotypiques entre cette variable et les 22 variables " descendants " sont faibles et aucune ne dépasse 0,08 en valeur absolue (les corrélations phénotypiques avec les variables $Y_{1}$ à $Y_{14}$ sont données dans le tableau IV 1).

TABLEAU 4

Héritabilités des variables "descendants".

Heritabilities of the progeny variables.

\begin{tabular}{|c|c|c|c|c|c|}
\hline \multicolumn{2}{|c|}{$\begin{array}{l}\text { Variables de croissance } \\
\text { et de carcasse } \\
\text { Growth and carcass traits }\end{array}$} & $h^{2} \pm$ e.s. & \multicolumn{2}{|c|}{$\begin{array}{c}\text { Variables " qualité de viande" } \\
\text { Meat quality traits }\end{array}$} & \multirow[t]{2}{*}{$h^{2} \pm$ e.s. } \\
\hline GMQ $(A D G) \ldots \ldots \ldots \ldots$ & $\mathrm{Y}_{1}$ & $0,30 \pm 0,07$ & $\mathrm{pH}$ & & \\
\hline Poids net (carcass weight) & $Y_{2}$ & $0,32 \pm 0,07$ & - Adductor femoris & $Y_{15}$ & $0,30 \pm 0,07$ \\
\hline Tête (head) & $\mathrm{Y}_{3}$ & $0,47 \pm 0,07$ & - Biceps femoris & $Y_{16}$ & $0,30 \pm 0,07$ \\
\hline Jambon (ham) .. & $\mathrm{Y}_{4}$ & $0,41 \pm 0,07$ & - Gluteus superficialis & $\mathrm{Y}_{17}$ & $0,21 \pm 0,06$ \\
\hline Longe (loin) $\ldots$. & $\mathrm{Y}_{5}$ & $0,62 \pm 0,08$ & - Longissimus dorsi. & $\mathrm{Y}_{18}$ & $0,15 \pm 0,06$ \\
\hline Poitrine (belly) & $Y_{6}$ & $0,29 \pm 0,07$ & & & \\
\hline Hachage (shoulder) & $\mathrm{Y}_{7}$ & $0,38 \pm 0,07$ & Reflectance $(r$ & & \\
\hline Pieds (legs) & $Y_{8}$ & $0,34 \pm 0,07$ & - Biceps femoris .... & $\begin{array}{l}Y_{19} \\
Y\end{array}$ & $\begin{array}{l}0,54 \pm 0,10 \\
020+0,06\end{array}$ \\
\hline Bardière (backfat) .. & $\mathrm{Y}_{9}$ & $0,90 \pm 0,09$ & - Urateus superfictats & 120 & $0,20 \perp 0,00$ \\
\hline Panne (leaf fat) $\ldots$. & $Y_{10}$ & $0,70 \pm 0,09$ & Temps d'imbilition & & \\
\hline Longueur (carcass length) & $Y_{11}$ & $0,21 \pm 0,06$ & (time to get wet) & & \\
\hline Lard (fat depth) & & & - Biceps femoris & $Y_{21}$ & $0,03 \pm 0,09$ \\
\hline - Rein (rump) & $Y_{12}$ & $0,80 \pm 0,08$ & - Gluteus superficialis & $\mathrm{Y}_{22}$ & $0,16 \pm 0,05$ \\
\hline - Dos (back) & $Y_{13}$ & $0,87 \pm 0,09$ & & & \\
\hline - Cou (shoulder) & $Y_{14}$ & $0,50 \pm 0,08$ & & & \\
\hline
\end{tabular}

C. Corrélations génétiques entre les variables "verrats" et les variables "descendants"

Les 44 corrélations obtenues, avec leur erreur-standard appoximative, figurent au tableau 5. La corrélation génétique élevée entre $X_{1}$ et $Y_{1}$ indique que la vitesse de croissance mesurée sur le verrat entre 30 et $80 \mathrm{~kg}$ est un bon critère de sélection pour améliorer la vitesse de croissance jusqu'au poids de $100 \mathrm{~kg}$ sur des femelles et des mâles castrés. La même observation vaut pour l'épaisseur de lard à $80 \mathrm{~kg}$ $\left(\mathrm{X}_{3}\right)$ qui est un bon prédicteur génétique des poids de longe et de bardière à $100 \mathrm{~kg}$ $\left(\mathrm{Y}_{5}\right.$ et $\left.\mathrm{Y}_{9}\right)$. Les corrélations génétiques concernant les mesures de $\mathrm{pH}$ et de réflectance sont, en moyenne, plus défavorables pour $X_{1}$ que pour $X_{3}$, l'inverse étant observé pour les temps d'imbibition. 


\section{TABLEAU 5}

Corrélations génétiques ( \pm e.s.) entre les variables * verrats " et les variables " descendants * (définies dans les tableaux 2 et 4 ).

Genetic correlations ( \pm s.e.) between boar and progeny variables

(defined in tables 2 and 4).

\begin{tabular}{|c|c|c|}
\hline \multirow{2}{*}{$\begin{array}{c}\text { Variable « descendants" } \\
\text { Progeny variable }\end{array}$} & \multicolumn{2}{|c|}{ Variable " verrats"-Boar variable } \\
\hline & $X_{1}$ & $\mathrm{X}_{3}$ \\
\hline$Y_{1} \quad \ldots$ & $1,13 \pm 0,16$ & $-0,05 \pm 0,14$ \\
\hline $\mathrm{Y}_{2} \ldots$ & $0,03 \pm 0,20$ & $0,10 \pm 0,14$ \\
\hline $\mathrm{Y}_{3} \ldots$ & $-0,19 \pm 0,18$ & $-0,58 \pm 0,10$ \\
\hline $\mathrm{Y}_{4} \ldots$ & $-0,12 \pm 0,19$ & $-0,63 \pm 0,10$ \\
\hline$Y_{5} \ldots$ & $-0,40 \pm 0,16$ & $-0,92 \pm 0,05$ \\
\hline$Y_{6} \ldots$ & $0,45 \pm 0,19$ & $0,45 \pm 0,12$ \\
\hline$Y_{7} \ldots$ & $-0,36 \pm 0,18$ & $-0,57 \pm 0,10$ \\
\hline$Y_{8} \quad \ldots$ & $-0,40 \pm 0,19$ & $-0,25 \pm 0,13$ \\
\hline $\mathrm{Y}_{9} \quad \ldots$ & $0,26 \pm 0,16$ & $0,84 \pm 0,06$ \\
\hline$Y_{10} \ldots$ & $-0,96 \pm 0,14$ & $0,61 \pm 0,08$ \\
\hline$Y_{11} \ldots$ & $-0,12 \pm 0,23$ & $-0,29 \pm 0,15$ \\
\hline $\mathrm{Y}_{12} \ldots$ & $0,11 \pm 0,16$ & $0,83 \pm 0,06$ \\
\hline $\mathbf{Y}_{13} \ldots$ & $0,09 \pm 0,16$ & $0,75 \pm 0,07$ \\
\hline$Y_{14} \ldots$ & $-0,05 \pm 0,18$ & $0,66 \pm 0,09$ \\
\hline $\mathrm{Y}_{15} \ldots$ & $-0,22 \pm 0,22$ & $0,15 \pm 0,15$ \\
\hline$Y_{16} \ldots$ & $-0,21 \pm 0,23$ & $0,07 \pm 0,15$ \\
\hline$Y_{17} \ldots$ & $0,24 \pm 0,24$ & $0,12 \pm 0,17$ \\
\hline$Y_{18} \ldots$ & $-0,02 \pm 0,28$ & $-0,04 \pm 0,18$ \\
\hline$Y_{19}$ & $0,22 \pm 0,20$ & $0,16 \pm 0,14$ \\
\hline$Y_{20} \ldots$ & $0,13 \pm 0,25$ & $-0,09 \pm 0,17$ \\
\hline$Y_{21}$ & $0,05 \pm 0,52$ & $0,49 \pm 0,43$ \\
\hline$Y_{22}$ & $0,03 \pm 0,27$ & $0,52 \pm 0,16$ \\
\hline
\end{tabular}

\section{Discussion et conclusions}

La structure des données de l'expérience qui vient d'être analysée présente des particularités rendant l'estimation des paramètres génétiques plus complexe que dans les situations habituellement rencontrées pour des caractères similaires dans l'espèce porcine. Les données permettent en effet d'estimer le même paramètre génétique de plusieurs façons et la question de l'estimation combinée se pose donc. C'est probablement Hazel (1943) qui a le premier rencontré cette situation dans le cas de la covariance géné- 
tique entre deux caractères mesurés sur un ensemble de couples parent-descendant. Mais l'estimateur de HazEL, qui est une moyenne arithmétique (ou géométrique), ne convient pas dans le cas de sélection (ou d'homogamie) des parents, et REeve (1953) a proposé une estimation combinée basée sur la méthode des moindres carrés. Plus récemment, des méthodes générales d'estimation combinée ont été proposées par EISEN (1967), Hill \& Nicholas (1974), Thompson (1977) et Gianola (1979), qui font appel à la méthode des moindres carrés ou à celle du maximum de vraisemblance. C'est la première de ces méthodes et la démarche générale proposée par Gianola qui a été retenue ici.

Il a fallu cependant, à chacune des trois étapes indiquées au chapitre Matériel et méthodes, apporter des simplifications afin de ne pas alourdir exagérément les calculs. (1) Dans l'estimation des covariances entre apparentés (D et G), le modèle mixte appliqué suppose l'absence d'interaction entre le facteur père et les facteurs de milieu considérés, ce qui peut avoir entraîné une surestimation des variances génétiques. (2) La correction pour les effets de la sélection ne tient pas compte de ses variations d'intensité au cours de l'expérience (voir Ollivier, 1977). C'est l'effet moyen de la sélection des verrats sur l'ensemble des générations qui est pris en compte, la population dont sont tirés les verrats étant elle-même considérée comme assez grande pour exclure une réduction appréciable de variabilité génétique au cours de l'expérience. On suppose par ailleurs une sélection purement massale sur $X_{1}$ (vitesse de croissance) et $X_{3}$ (épaisseur de gras) et la correction ne prend donc pas en considération la sélection sur $\mathrm{X}_{2}$ (indice de consommation) effectuée au cours des trois premières générations ni la méthode de sélection, intra-famille de père, appliquée jusqu'en 1971. On a de plus admis que les verrats fondateurs ont subi le même type de sélection que ceux de l'expérience, ce qui peut fausser les corrections appliquées aux variances et covariances entre demi-germains pour $\mathbf{X}^{\prime}$ et $\mathbf{Y}\left(\mathbf{G}_{x^{\prime} x^{\prime}}\right.$ et $\left.\mathbf{G}_{y y}\right)$. (3) La méthode d'estimation combinée qui est détaillée dans l'annexe I ne s'applique en toute rigueur qu'aux estimateurs définis en (1) et ne prend donc pas en compte les corrections (2) pour les effets de la sélection. La procédure elle-même repose par ailleurs sur de nombreuses hypothèses statistiques. Rappelons d'abord la multinormalité des distributions - équations (I 1) - situation qui est approximativement vérifiée pour toutes les variables sauf $\mathrm{Y}_{21}$ et $\mathrm{Y}_{22}$ (temps d'imbibition) dont les distributions sont fortement dissymétriques. Notons aussi que les équations (I 1) supposent que les pères soient indépendants entre eux, une condition non remplie dans cette étude qui porte sur dix générations paternelles successives. A cela s'ajoutent les approximations dans le calcul des variances d'échantillonnage des héritabilités et corrélations génétiques - équations (II 1) et (III 1) - et celles qui résultent des inégalités d'effectif par père et par mère, dont la planification de l'expérience tend cependant à atténuer les effets. Pour toutes ces raisons, les variances d'échantillonnage des tableaux $3,4,5$ sont à considérer comme des sousestimations des valeurs réelles, mais il n'y a pas de raison de penser que les combinaisons d'estimateurs elles-mêmes, c'est-à-dire les coefficients $\boldsymbol{\alpha}, \boldsymbol{\beta}$ et $\boldsymbol{\gamma}$ des équations (II 2), en soient notablement affectées. Enfin, du point de vue génétique, il faut rappeler que l'estimation combinée suppose que les covariances $\mathbf{D}$ et $\mathbf{G}$ soient fonctions seulement des variances et covariances génétiques additives, ce qui implique l'absence d'épistasie (mais non de dominance) ainsi que de tout effet maternel ou de milieu sur les covariances estimées. C'est la raison pour laquelle la relation entre germains n'a pas été utilisée, au prix de l'élimination d'une partie des données disponibles pour le calcul de $\mathbf{G}_{x^{\prime} y}$ (voir tableau 1). Par ailleurs, l'effet de l'élevage de naissance des individus sur les covariances entre apparentés peut ici être considéré comme négligeable, l'insémination artificielle assurant une distribution aléatoire des pères dans les élevages. 
Le tableau 2 permet de juger, pour les variables "verrats ", de la cohérence des estimées de la covariance parent-descendant ( $\hat{D})$ et de la covariance entre demigermains $(\hat{G})$ pour chaque combination de variables. Les rapports observés entre ces covariances sont proches de la valeur $2: 1$ attendue, mis à part le cas, déjà mentionné plus haut, des covariances entre $X_{1}$ et $X^{\prime}{ }_{1}$ d'une part, et entre $X^{\prime}{ }_{1}$ d'autre part, entre lesquelles le rapport est plus élevé, tout en étant compatible avec la valeur attendue compte tenu de la précision des estimations. Les estimées élémentaires des covariances génétiques entre les variables «verrats " et les variables "descendants " (non données ici) montrent des tendances similaires, la covariance génétique dérivée de la relation parent-descendant étant généralement proche de celle déduite de la relation entre demi-germains, sauf pour la combinaison $X_{1} Y_{1}$ où la première estimation est nettement supérieure à la deuxième. La procédure d'estimation combinée paraît donc justifiée pour l'ensemble des paramètres génétiques considérés, à l'exception peut-être de la variance génétique de $X_{1}$ et de la covariance génétique entre $X_{1}$ et $Y_{1}$.

Les valeurs d'héritabilités des tableaux 3 et 4 confirment les nombreux résultats publiés dans la littérature : valeurs moyennes pour les caractères de croissance $\left(\mathrm{X}_{1}\right.$ et $\left.\mathrm{Y}_{1}\right)$ et de qualité de viande (à l'exception cependant des temps d'imbibition $\mathrm{Y}_{21}$ et $\mathrm{Y}_{22}$ ), valeurs élevées pour les mesures de carcasse et tout particulièrement pour les critères d'adiposité $\left(\mathrm{X}_{3}, \mathrm{Y}_{9}, \mathrm{Y}_{10}, \mathrm{Y}_{12}, \mathrm{Y}_{13}\right)$. Notons en particulier, pour les caractères sélectionnés $X_{1}$ et $X_{3}$, que des héritabilités voisines de celles du tableau 3 ont été obtenues dans deux expériences de sélection similaires à celle-ci, sur la base de la covariance entre demi-germains mais sans correction pour les effets de la sélection (WebB \& KING, 1976 ; V ANGEN, 1979). Par ailleurs, Webb et King obtiennent pour la vitesse de croissance une héritabilité nettement plus élevée à partir de la relation mère-fille, ce qui va dans le sens des résultats du tableau 2. Par contre, la corrélation génétique fortement positive trouvée entre $X_{1}$ et $X_{3}$ contraste avec la faible valeur donnée par Vangen et elle paraît d'autre part peu compatible avec les autres corrélations génétiques entre croissance et adiposité des tableaux 5 et IV 1, qui sont dans l'ensemble faibles.

L'information génétique essentielle, dans le contexte de l'expérience de sélection analysée, est donnée dans le tableau 5. En fait l'efficacité de la sélection dépend des corrélations (ou des covariances) génétiques entre les caractères $\mathrm{X}$ mesurés sur les verrats et les caractères $\mathrm{Y}$ mesurés sur leur descendance. Une situation analogue est celle où $\mathrm{X}$ et $\mathrm{Y}$ sont les mêmes caractères mesurés dans des conditions de milieu différentes, par exemple en station de sélection et en élevage, auquel cas la corrélation génétique est une mesure de l'importance de l'interaction génotype-milieu. Les études réalisées en Norvège (Standal, 1977) et en Grande-Bretagne (Bampton et al, 1977 ; Roberts \& Curran, 1981) montrent que les corrélations génétiques entre des caractères similaires mesurés en station et en élevage sont généralement très inférieures à 1 , ce qui résulte de différences dans les conditions d'alimentation, les méthodes de mesure, les périodes de contrôle, et aussi le sexe des individus. Des interactions génotype $\times$ sexe ont, en particulier, été mises en évidence en station par SMITH \& Ross (1965), mais n'ont pas été confirmées depuis, comme le remarque Standal (1977). Il est par ailleurs difficile de dissocier la part de ce facteur dans les comparaisons station-élevage. Par contre, dans l'expérience analysée ici, les conditions de milieu sont les mêmes pour $\mathrm{X}$ et pour $\mathrm{Y}$, les seules différences concernant le sexe des animaux et la période de contrôle de croissance. La corrélation élevée entre $X_{1}$ et $Y_{1}$ suggère donc l'absence d'interaction génotype-sexe ou génotype - période de contrôle pour la vitesse de croissance, et la même remarque vaut pour les critères d'adiposité mesurés sur le verrat ou sur l'animal abattu et découpé. Le système d'alimentation ad libitum ne permettant pas de mesurer 
les consommations individuelles, il n'est pas possible d'estimer les corrélations génétiques relatives à l'indice de consommation. L'estimation des paramètres génétiques réalisés, qui fera l'objet d'un article ultérieur, doit permettre de combler cette lacune, tout en complétant l'information sur l'ensemble des corrélations génétiques.

Reçu pour publication le 23 août 1982. Accepté pour publication le 4 novembre 1982.

\section{Remerciements}

L'auteur est reconnaissant à ceux qui ont assuré la lecture critique de ce manuscrit pour leurs observations.

\section{Références bibliographiques}

Aitken A.C., 1934. Note on selection from a multivariate normal population. Proc. Edinburgh Math. Soc., 4, 106-110.

Bampton P.R., Curran M.K., Kempson R.E., 1977. A comparison of " on farm" and station testing in pigs. Anim. Prod., 25, 83-94.

EISEN E.J., 1967. Mating designs for estimating direct and maternal genetic variances and direct-maternal genetic covariances. Can J. Genet. Cytol., 9, 13-22.

Gianola D., 1979. Estimation of genetic covariance from joint offspring-parent and sib-sib statistics. Genetics, 93, 1039-1049.

HARVEY W.R., 1972. Least-squares and maximum likelihood general purpose programme. Ohio State University.

Hazel L.N., 1943. The genetic basis for constructing selection indexes. Genetics, 28, 476-490.

Hill W.G., Nicholas F.W., 1974. Estimation of heritability by both regression of offspring on parent and intra-class correlation of sibs in one experiment. Biometrics, 30, 447-468.

Kendall M.G., Stuart A., 1958. The advanced theory of statistics, vol. 1, Griffin, Londres.

Ollivier L., 1977. Dix ans d'une expérience de sélection individuelle sur des verrats utilisés en insémination artificielle. I - Réponses observées sur des caractères de croissance de carcasse et de qualité de viande. Ann. Génét. Sél. anim., 9, 353-377.

Ollivier L., Derrien A., 1981. Une méthode générale d'estimation des paramètres génétiques dans un échantillon sélectionné, avec une application à une sélection sur un indice à 3 caractères. Ann. Génét. Sél. anim., 13, 281-292.

REEVE E.C.R., 1953. Studies in quantitative inheritance. III - Heritability and genetic correlation in progeny tests using different mating systems. J. Genet., 51, 520-542.

Roberts D.J., CuRran M.K., 1981. A comparison of " on farm » and station testing in pigs. Anim. Prod., 33, 291-297.

SEarle S.R., 1971. Linear Models. John Wiley and Sons, New York.

Sмiтн C., Ross G.J.S., 1965. Genetic parameters of British Landrace bacon pigs. Anim. Prod., 7, 291-301.

Standal N., 1977. Studies on breeding and selection schemes in pigs. VI - Correlation between breeding values estimated from station test and on-farm test data. Acta agric. scand., 27, 138-144. 
THOMPson R., 1977. Estimation of quantitative genetic parameters. In : Pollak E., KempThORNe O., Bailey T.B. Jr., Proceedings of the International Conference on Quantitative Genetics, 639-657. The Iowa State University Press, Ames, Iowa.

VANGEN O., 1979. Studies on a two trait selection experiment in pigs. II - Genetic changes and realized genetic parameters in the traits under selection. Acta agric. scand., 29, 305-319.

WebB A.J., King J.W.B., 1976. Development of a synthetic pig sire line by selection with immigration. I - Results of selection and heritability estimates. Anim Prod., 22, 231-244.

\section{Annexe I}

Eléments nécessaires à l'estimation combinée des variances et covariances génétiques relatives à des caractères $X$ mesurés sur deux générations successives et à des caractères $Y$ mesurés sur une seule génération

Le dispositif expérimental est constitué de pères (en nombre p) mesurés pour un ensemble de caractères $\mathrm{X}$ et de deux échantillons de descendants de ces pères, mesurés l'un pour $\mathrm{X}$ et l'autre pour un autre ensemble de caractères Y. Pour simplifier nous supposons d'abord que $X$ est mesuré sur un nombre constant de descendants par père, répartis entre $m$ mères et $n$ descendants par mère, et que $Y$ est mesuré sur $m^{\prime} n^{\prime}$ descendants par père selon une répartition analogue, l'échantillon des pm mères (pour $\mathrm{X}$ ) étant distinct de celui des $\mathrm{pm}^{\prime}$ mères (pour Y). Dans un tel dispositif, deux estimations différentes de la variance génétique d'un caractère $X$ peuvent être obtenues, l'une à partir de la covariance parent-descendant (désignée par $\hat{\mathrm{D}}_{\mathrm{xx}}$ ) et l'autre à partir de la covariance entre demi-germains (désignée par $\hat{\mathrm{G}}_{\mathrm{xx}}$ ). La covariance génétique entre 2 caractères $X$ quelconques, $x$ et $x^{*}$, peut être obtenue de trois façons, puisque à la covariance entre demi-germains $\left(\hat{\mathrm{G}}_{\mathrm{xx}^{*}}\right)$ s'ajoutent deux covariances parent-descendant $\left(\hat{D}_{x^{*}}\right.$ et $\left.\hat{D}_{x^{*} x}\right)$, selon la variable attribuée au parent. Quant aux covariances entre $X$ et $\mathrm{Y}$, deux estimateurs existent, dérivés de $\hat{D}_{x y}$ et de $\hat{G}_{x y}$. Le premier se calcule comme $\hat{\mathrm{D}}_{\mathrm{xx}}$ - à partir de la régression parent-descendant ou du coproduit entre le parent et la moyenne de ses descendants - mais $\hat{G}_{x y}$ est un estimateur différent de $\hat{G}_{x x}$, celui-ci étant la composante paternelle de variance tirée de l'analyse de variance classique du dispositif père-mère-résiduelle alors que $\hat{G}_{x y}$ est le coproduit entre les moyennes de descendance de $\mathrm{X}$ et de $\mathrm{Y}$. Précisons que, dans la notation retenue ici, le premier indice de $D$ désigne la variable mesurée sur le parent. Ainsi $\hat{D}_{x x}$ est-il l'élément relatif à une variable $X$ de $\mathbf{D}_{x x^{\prime}}$ défini en (1). De même $\hat{G}_{x x}$ correspond à $\mathbf{G}_{x^{\prime} x^{\prime}}$ et $\hat{G}_{x y}$ à $\mathbf{G}_{x^{\prime} y}$.

Les estimations combinées $(\mathrm{E})$ des variances et covariances génétiques sont obtenues par la méthode des moindres carrés généralisés, qui requiert le calcul des variances (V) et covariances (Cov) des différents estimateurs. Celles-ci s'écrivent à partir de la formule générale de la covariance entre formes bilinéaires, selon une procédure, décrite par Gianola (1979), qui va être brièvement expliquée. Prenons le cas de quatre variables aléatoires - avec une distribution quadrivariate normale de moyennes quelconques et de variances-covariances désignées par $\sigma-$ auxquelles correspondent quatre vecteurs, $1,2,3,4$, représentant le résultat de $\mathrm{p}$ tirages indépendants des quatre variables. En exprimant chaque élément de ces vecteurs en écart à la moyenne de la variable concernée sur les p tirages, les variables considérées 
ont des espérances nulles, et la covariance entre deux formes bilinéaires quelconques, 1'3 et 2' 4 par exemple, se déduit de la formule générale (voir, par exemple, Searle, 1971, p. 66) :

$$
\operatorname{Cov}\left(1^{\prime} 3,2^{\prime} 4\right)=\operatorname{tr}\left(\mathbf{C}_{14}^{\prime} \mathbf{C}_{23}^{\prime}+\mathbf{C}_{12} \mathbf{C}_{34}\right)
$$

où tr désigne l'opérateur trace d'une matrice, soit la somme de ses termes diagonaux, et les C sont des matrices de covariance entre les éléments, considérés deux à deux, des vecteurs désignés en indice. $\mathbf{C}_{14}$ est ainsi le produit de $\sigma_{14}$, la covariance entre les variables 1 et 4 , par une matrice dont les $p$ termes diagonaux sont tous égaux à $1-1 / p$ et les termes hors-diagonale à $-1 / p$. En explicitant les deux produits matriciels ci-dessus il est facile de montrer que la covariance entre les deux estimateurs de covariance $\hat{\sigma}_{13}=\left(1^{\prime} 3\right) /(p-1)$ et $\hat{\sigma}_{24}=\left(2^{\prime} 4\right) /(p-1)$ s'écrit :

$$
\operatorname{Cov}\left(\hat{\sigma}_{13}, \hat{\sigma}_{24}\right)=\left(\sigma_{14} \sigma_{23}+\sigma_{12} \sigma_{34}\right) /(\mathrm{p}-1)
$$

Cette expression générale peut s'appliquer aux estimateurs définis plus haut, qui sont en effet soit des formes bilinéaires simples (cas des D) soit des combinaisons linéaires de coproduits moyens (cas des $\hat{G}$ ), ceux-ci étant eux-mêmes des formes bilinéaires. Dans le cas de quatre caractères différents mesurés sur les parents et sur les descendants, il vient :

$$
\begin{aligned}
& \operatorname{Cov}\left(\hat{\mathrm{D}}_{13}, \hat{\mathrm{D}}_{24}\right)=\left(\mathrm{D}_{14} \mathrm{D}_{23}+\sigma_{12} \lambda_{34}\right) /(\mathrm{p}-1) \\
& \operatorname{Cov}\left(\hat{\mathrm{D}}_{13}, \mathrm{G}_{24}\right)=\left(\mathrm{D}_{14} \lambda_{23}+\mathrm{D}_{12} \lambda_{34}\right) /(\mathrm{p}-1) \\
& \operatorname{Cov}\left(\widehat{\mathrm{PM}}_{13}, \widehat{\mathrm{PM}}_{24}\right)=\left(\mathrm{PM}_{14} \mathrm{PM}_{23}+\mathrm{PM}_{12} \mathrm{PM}_{34}\right) / \mathrm{d}
\end{aligned}
$$

où $\sigma_{12}$ est la covariance phénotypique entre 1 et 2 (on posera $\sigma_{11}=\sigma_{1}^{2}=$ variance de 1 );

$\lambda_{34}=\sigma_{34}\left[1+(n-1) t_{1}+n(m-1) t_{2}\right] / m n, t_{1}$ et $t_{2}$ étant les covariances respectives entre germains et demi-germains rapportées à la covariance totale entre 3 et 4 (on posera $\lambda_{11}=\lambda_{1}^{2}=$ variance de la moyenne des descendants pour 1);

PM désigne des coproduits moyens et d les degrés de liberté correspondants dans l'analyse de covariance (CM désignera les carrés moyens) ;

les $\mathrm{D}, \mathrm{PM}, \sigma$ et $\lambda$ sont les vraies valeurs dans la population.

Par ailleurs, dans le dispositif décrit ci-dessus, les composantes de variance paternelle $\left(\sigma_{\mathrm{p}}^{2}\right)$, maternelle $\left(\sigma_{\mathrm{M}}^{2}\right)$ et résiduelle $\left(\sigma_{\mathrm{R}}^{2}\right)$ de $\mathrm{X}$ s'écrivent en fonction des carrés moyens correspondants, $\widehat{\mathrm{CM}}_{\mathrm{P}}, \widehat{\mathrm{CM}}_{\mathrm{M}}, \widehat{\mathrm{CM}}_{\mathrm{R}}$ :

$$
\begin{aligned}
& \hat{\sigma}_{\mathrm{P}}^{2}=\hat{\mathrm{G}}=\left(\widehat{\mathrm{CM}}_{\mathrm{P}}-\widehat{\mathrm{CM}}_{\mathrm{M}}\right) / \mathrm{mn} \\
& \hat{\sigma}_{\mathrm{M}}^{2}=\left(\widehat{\mathrm{CM}}_{\mathrm{M}}-\widehat{\mathrm{CM}}_{\mathrm{R}}\right) / \mathrm{n} \\
& \hat{\sigma}_{\mathrm{R}}^{2}=\widehat{\mathrm{CM}}_{\mathrm{R}}
\end{aligned}
$$

et on a : $t_{1}=\left(\sigma_{\mathrm{p}}^{2}+\sigma_{\mathrm{M}}^{2}\right) / \sigma^{2}$ et $t_{2}=\sigma_{\mathrm{p}}^{2} / \sigma^{2}$. Des expressions analogues s'écrivent avec les composantes de covariance.

Les équations (I l) permettent d'écrire, en tenant compte de (I 2) pour le calcul de $\mathrm{V}\left(\hat{\mathrm{G}}_{\mathrm{xx}}\right)$ :

- Pour $E_{x x}$

$$
\begin{aligned}
& \mathrm{V}\left(\hat{\mathrm{D}}_{\mathrm{xx}}\right)=\left(\mathrm{D}_{\mathrm{xx}}^{2}+\sigma_{\mathrm{x}}^{2} \lambda_{\mathrm{x}}^{2}\right) /(\mathrm{p}-1) \\
& V\left(\hat{G}_{\mathrm{xx}}\right)=\frac{2}{(\mathrm{mn})^{2}}\left[\mathrm{CM}_{\mathrm{p}}^{2}+(\mathrm{p}-1) \mathrm{CM}_{\mathrm{M}}^{2} / \mathrm{p}(\mathrm{m}-1)\right] /(\mathrm{p}-1) \\
& \operatorname{Cov}\left(\hat{\mathrm{D}}_{\mathrm{xx}}, \hat{\mathrm{G}}_{\mathrm{xx}}\right)=2 \mathrm{D}_{\mathrm{xx}} \lambda_{\mathrm{x}}^{2} /(\mathrm{p}-1)
\end{aligned}
$$


- Pour $E_{x x^{*}}$

$$
\left.\begin{array}{l}
\left.\mathrm{V} \hat{\mathrm{D}}_{\mathrm{xx}^{*}}\right)=\left(\mathrm{D}_{\mathrm{xx^{* }}}^{2}+\sigma_{\mathrm{x}}^{2} \lambda_{\mathrm{x}^{*}}^{2}\right) /(\mathrm{p}-1) \\
\operatorname{Cov}\left(\hat{\mathrm{D}}_{\mathrm{xx^{* }}}, \hat{\mathrm{G}}_{\mathrm{xx}^{*}}\right)=\left(\mathrm{D}_{\mathrm{xx}{ }^{*}} \lambda_{\mathrm{xx}^{*}}+\mathrm{D}_{\mathrm{xx}} \lambda_{\mathrm{x}^{*}}^{2}\right) /(\mathrm{p}-1) \\
\operatorname{Cov}\left(\hat{\mathrm{D}}_{\mathrm{xx}^{*}}, \hat{\mathrm{D}}_{\mathrm{x}^{*} \mathrm{x}}\right)=\left(\mathrm{D}_{\mathrm{xx}} \mathrm{D}_{\mathrm{x}^{*} \mathrm{x}^{*}}+\sigma_{\mathrm{xx}} \lambda_{\mathrm{xx}}\right) /(\mathrm{p}-1)
\end{array}\right\}
$$

sachant que $\mathrm{V}\left(\hat{\mathrm{D}}_{\mathrm{x}^{*} \mathrm{x}}\right)$ et $\operatorname{Cov}\left(\hat{\mathrm{D}}_{\mathrm{x}^{*} \mathrm{x}}, \hat{\mathrm{G}}_{\mathrm{xx}^{*}}\right)$ sont obtenus en permutant $\mathrm{x}$ et $\mathrm{x}^{*}$ dans (I 4) et que $\mathrm{V}\left(\hat{\mathrm{G}}_{\mathrm{xx}}\right)$ a la même expression que $\mathrm{V}\left(\hat{\mathrm{G}}_{\mathrm{xx}}\right)$ avec substitution des coproduits aux carrés moyens correspondants.

- Pour $E_{x y}$

Sachant que $G_{x y}$ est une covariance entre deux variables dont les variances sont $\lambda_{x}^{2}$ et $\lambda_{y}^{2}$, il vient en application de (I 1$)$ :

$$
\left.\begin{array}{l}
V\left(\hat{D}_{x y}\right)\left(D_{x y}^{2}+\sigma_{x}^{2} \lambda_{y}^{2}\right) /(p-1) \\
V\left(\hat{G}_{x y}\right)=\left(\hat{G}_{x y}^{2}+\lambda_{x}^{2} \lambda_{y}^{2}\right) /(p-1) \\
\operatorname{Cov}\left(\hat{D}_{x y}, \hat{G}_{x y}\right)=\left(D_{x y} G_{x y}+D_{x x} \lambda_{y}^{2}\right) /(p-1)
\end{array}\right\}
$$

Les formules précédentes ne s'appliquent rigoureusement que si le dispositif est équilibré. GianOLA (1979) donne des expressions pour le cas d'effectifs inégaux par père et $\mathrm{n}=1$. Lorsque $\mathrm{n}$ est variable, l'approximation au schéma équilibré apporte une appréciable simplification et les formules précédentes sont alors modifiées conme suit :

$$
\begin{aligned}
& \mathrm{V}\left(\hat{\mathrm{G}}_{\mathrm{xx}}\right)=\frac{2}{\mathrm{k}_{1}^{2}}\left[\left(\mathrm{CM}_{\mathrm{P}}^{2} / \mathrm{d}_{\mathrm{P}}\right)+\left(\mathrm{k}_{2} / \mathrm{k}_{3}\right)^{2}\left(\mathrm{CM}_{\mathrm{M}}^{2} / \mathrm{d}_{\mathrm{M}}\right)+\left(1-\mathrm{k}_{2} / \mathrm{k}_{3}\right)^{2} \mathrm{CM}_{\mathrm{R}}^{2} / \mathrm{d}_{\mathrm{R}}\right] \\
& \lambda^{2}=\sigma_{\mathrm{P}}^{2}+\left(\mathrm{k}_{3} / \mathrm{k}_{1}\right) \sigma_{\mathrm{M}}^{2}+\left(1 / \mathrm{k}_{1}\right) \sigma_{\mathrm{R}}^{2}
\end{aligned}
$$

où $d_{P}\left(d_{P}=p-1\right), d_{M}$ et $d_{R}$ sont les degrés de liberté correspondant à $C_{P}, C_{M}$ et $\mathrm{CM}_{\mathrm{R}}$ respectivement, $\mathrm{k}_{1}$ et $\mathrm{k}_{2}$ les coefficients de $\sigma_{\mathrm{P}}^{2}$ et $\sigma_{\mathrm{M}}^{2}$ dans l'espérance de $\mathrm{CM}_{\mathrm{P}}$ et $k_{3}$ le coefficient de $\sigma_{M}^{2}$ dans l'espérance de $C_{M}$. Les covariances entre carrés moyens sont négligées dans $\mathrm{V}\left(\hat{\mathrm{G}}_{\mathrm{x} x}\right)$ et l'expression de $\lambda^{2}$ suppose des effectifs constants de $k_{1}$ descendants par père et $k_{1} / k_{3}$ par mère.

\section{Annexe II}

Variances d'échantillonnage approximatives de corrélations génétiques basées sur des estimations combinées de variances et de covariances génétiques

La corrélation génétique entre 2 variables quelconques ( 1 et 2) s'écrit :

$$
\hat{\mathrm{r}}_{\mathrm{A}}=\hat{\mathrm{E}}_{12} /\left(\hat{\mathrm{E}}_{11} \hat{\mathrm{E}}_{22}\right)^{0.5}
$$

où $\hat{E}_{12}, \hat{E}_{11}$ et $\hat{E}_{22}$ sont les estimations combinées respectives des covariances génétiques et des variances génétiques relatives aux 2 variables considérées. La variance d'échantillonnage approchée est donnée par l'expression suivante (voir Kendall \& STUart, 1958 , p. 235) :

$$
\left.\begin{array}{l}
\frac{V\left(\hat{r}_{A}\right)}{r_{A}^{2}} \simeq\left[V\left(\hat{E}_{12}\right) / E_{12}^{2}\right]+\left[V\left(\hat{E}_{11}\right) / 4 E_{11}^{2}\right]+\left[V\left(\hat{E}_{22}\right) / 4 E_{22}^{2}\right] \\
\left.\left.E_{11}\right) / E_{12} E_{11}\right]-\left[\operatorname{Cov}\left(\hat{E}_{12}, \hat{E}_{22}\right) / E_{12} E_{22}\right]+\left[\operatorname{Cov}\left(\hat{E}_{11}, \hat{E}_{22}\right) / 2 E_{11} E_{22}\right]
\end{array}\right\}
$$


Les variances et les covariances du second membre de cette équation s'expriment en fonction de la matrice $\mathbf{C}$ des variances et covariances relatives aux estimateurs utilisés et des vecteurs $\boldsymbol{\alpha}, \boldsymbol{\beta}$ et $\boldsymbol{\gamma}$ des coefficients de pondération impliqués dans $\hat{\mathrm{E}}_{11}, \hat{\mathrm{E}}_{22}$ et $\hat{\mathrm{E}}_{12}$ respectivement, soit :

$$
\begin{aligned}
& \mathrm{V}\left(\hat{\mathrm{E}}_{11}\right)=\boldsymbol{\alpha}^{\prime} \mathbf{C} \boldsymbol{\alpha} \\
& \operatorname{Cov}\left(\hat{\mathrm{E}}_{11}, \hat{\mathrm{E}}_{12}\right)=\boldsymbol{\alpha}^{\prime} \mathbf{C} \boldsymbol{\gamma}, \text { etc. }
\end{aligned}
$$

\section{Cas de deux caractères $(X)$ mesurés sur les parents et les descendants}

Dans ce cas, $\boldsymbol{\alpha}$ est un vecteur à deux éléments non nuls correspondant à $\hat{D}_{x x}\left(\alpha_{1}\right)$ et $\hat{G}_{x x}\left(\alpha_{2}\right)$, de même $\beta$ est un vecteur à deux éléments non nuls correspondants à $\hat{D}_{x^{*} x^{*}}\left(\beta_{1}\right)$, et $\hat{G}_{x^{*} x^{*}}\left(\beta_{2}\right)$ et $\boldsymbol{\gamma}$ est un vecteur à trois éléments non nuls correspondant à $\hat{D}_{x^{*}}\left(\gamma_{1}\right), \hat{D}_{x^{*} x}\left(\gamma_{2}\right)$ et $\hat{G}_{x^{*}}\left(\gamma_{3}\right)$.

On a donc :

$$
\begin{aligned}
\boldsymbol{\alpha}^{\prime} & =\left(\begin{array}{lllllll}
\alpha_{1} & \alpha_{2} & 0 & 0 & 0 & 0 & 0
\end{array}\right) \\
\boldsymbol{\beta}^{\prime} & =\left(\begin{array}{llllllll}
0 & 0 & \beta_{1} & \beta_{2} & 0 & 0 & 0
\end{array}\right) \\
\boldsymbol{\gamma}^{\prime} & =\left(\begin{array}{lllllll}
0 & 0 & 0 & 0 & \gamma_{1} & \gamma_{2} & \gamma_{3}
\end{array}\right)
\end{aligned}
$$

Les éléments de $\mathbf{C}$ nécessaires au calcul de $\mathrm{V}\left(\hat{\mathrm{E}}_{11}\right), \mathrm{V}\left(\hat{\mathrm{E}}_{22}\right)$ et $\mathrm{V}\left(\hat{\mathrm{E}}_{12}\right)$ sont donnés par les équations (I 3) et (I 4). Mais les trois derniers termes de (II 1) font appel à des éléments de $\mathbf{C}$ qui restent à calculer. Ces éléments, obtenus à partir des formules générales (I 1), sont listés ci-dessous, chaque élément étant multiplié par $(p-1)$ et indicé par son numéro de ligne et de colonne :

$$
\begin{array}{ll}
C_{13}=D_{x x} D_{x^{*} x}+\sigma_{x x^{*}} \lambda_{x^{*}} & C_{23}=2 D_{x^{*} x} \lambda_{x^{*}} \\
C_{14}=2 D_{x^{*}} \lambda_{x x^{*}} & C_{24}=\frac{2}{(m n)^{2}}\left[P_{P}^{2}+P_{M}^{2}(p-1) / p(m-1)\right] \\
C_{15}=D_{x x^{*}} D_{x x}+\sigma_{x}^{2} \lambda_{x x^{*}} & C_{25}=2 D_{x x} \lambda_{x x^{*}} \\
C_{16}=D_{x x} D_{x^{*} x}+\sigma_{x x^{*}} \lambda_{x}^{2} & C_{26}=2 D_{x^{*} x} \lambda_{x}^{2} \\
C_{17}=D_{x x^{*}} \lambda_{x}^{2}+D_{x x} \lambda_{x x^{*}} & C_{27}=\frac{2}{(m n)^{2}}\left[C_{P} P_{P}+C M_{M} P_{M}(p-1) / p(m-1)\right]
\end{array}
$$

avec les notations définies dans l'annexe $I, C_{P}\left(C_{M}\right)$ et $\mathrm{PM}_{P}\left(\mathrm{PM}_{\mathrm{M}}\right)$ étant respectivement les carrés et coproduits moyens paternels (maternels) pour $\mathrm{x}$ et $\mathrm{xx}^{*}$.

Les termes $C_{35}, C_{36}$ et $C_{37}$ se déduisent respectivement de $C_{16}, C_{15}$ et $C_{17}$ en permutant $\mathrm{x}^{*}$ et $\mathrm{x}$ et de même pour les termes $\mathrm{C}_{45}, \mathrm{C}_{46}$ et $\mathrm{C}_{47}$ à partir de $\mathrm{C}_{26}$, $\mathrm{C}_{25}$ et $\mathrm{C}_{27}$.

Lorsque le dispositif est déséquilibré, les éléments de $\mid \mathbf{C}$ indiqués ci-dessus ne sont plus rigoureusement applicables. L'approximation au schéma équilibré permet de calculer les $\lambda$ et les éléments $C_{24}$ et $C_{27}$ en fonction des coefficients $k_{1}, k_{2}$ et $k_{3}$ et des degrés de liberté $d_{P}, d_{M}$ et $d_{R}$, comme indiqué dans l'annexe $I$. 
Cas d'un caractère $(X)$ mesuré sur les parents et les descendants et d'un caractère $(Y)$ mesuré seulement sur les descendants

Les expressions générales (II 1) et (II 2) s'appliquent à ce cas, en posant $1=x$ et $2=y$, à condition de redéfinir les éléments non nuls de $\boldsymbol{\beta}$ et $\boldsymbol{\gamma}$. Comme $\hat{\mathrm{E}}_{22}=\hat{\mathrm{E}}_{\mathrm{yy}}=4 \hat{\mathrm{G}}_{\mathrm{yy}}$ et que $\hat{\mathrm{E}}_{12}=\hat{\mathrm{E}}_{\mathrm{xy}}$ est seulement basé sur deux estimateurs $\hat{\mathrm{D}}_{\mathrm{xy}}$ et $\hat{\mathrm{G}}_{\mathrm{xy}}$ dont les coefficients respectifs sont $\gamma_{1}$ et $\gamma_{2}$, on a :

$$
\begin{aligned}
& \boldsymbol{\alpha}^{\prime}=\left(\begin{array}{lllll}
\alpha_{1} & \alpha_{2} & 0 & 0 & 0
\end{array}\right) \\
& \boldsymbol{\beta}^{\prime}=\left(\begin{array}{lllll}
0 & 0 & 4 & 0 & 0
\end{array}\right) \\
& \boldsymbol{\gamma}^{\prime}=\left(\begin{array}{lllll}
0 & 0 & 0 & \gamma_{1} & \gamma_{2}
\end{array}\right)
\end{aligned}
$$

Les éléments de la matrice $\mathbf{C}$ nécessaires au calcul des trois derniers termes de (II 1) sont, avec les mêmes conventions que précédemment, et sachant que $\lambda_{\mathrm{xy}}=\mathrm{G}_{\mathrm{xy}}$ :

$$
\begin{array}{lll}
C_{13}=2 D_{x y} G_{x y} & C_{14}=D_{x y} D_{x x}+\sigma_{x}^{2} G_{x y} & C_{15}=D_{x y} \lambda_{x}^{2}+D_{x x} G_{x y} \\
C_{23}=2 G_{x y}^{2} & C_{24}=2 D_{x x} G_{x y} & C_{25}=2 G_{x y} \lambda_{x}^{2} \\
C_{34}=2 D_{x y} \lambda_{y}^{2} & C_{35}=2 G_{x y} \lambda_{y}^{2} &
\end{array}
$$

Dans le cas d'un dispositif déséquilibré, l'approximation donnée pour $\lambda$ dans l'annexe I peut être utilisée.

\section{Annexe III}

Variance d'échantillonnage approximative d'une héritabilité basée sur une estimation combinée de la variance génétique

L'héritabilité est estimée par le rapport :

$$
\hat{\mathrm{h}}^{2}=\mid \hat{\mathrm{E}} /\left(0,25 \hat{\mathrm{E}}+\hat{\sigma}_{\mathrm{M}}^{2}+\hat{\sigma}_{\mathrm{R}}^{2}\right)=\hat{\mathrm{E}} / \hat{\sigma}^{2}
$$

où $\hat{E}$ est la variance génétique additive estimée par une combinaison linéaire de deux covariances $\hat{D}$ et $\hat{\mathbf{G}}$, telle que :

$$
\hat{\mathrm{E}}=\alpha_{1} \hat{\mathrm{D}}+\alpha_{2} \hat{\mathrm{G}}
$$

$\hat{\mathrm{D}}, \hat{\mathrm{G}}, \hat{\sigma}_{\mathrm{M}}^{2}$ et $\hat{\sigma}_{\mathrm{R}}^{2}$ étant définis dans l'annexe $\mathbf{I}$.

Une variance d'échantillonnage approchée de $\hat{h}_{2}$ est calculée selon le même principe que pour $\hat{\mathrm{r}}_{\mathrm{A}}$ (annexe II) et on obtient :

$$
\left.\begin{array}{c}
\sigma^{8} V\left(\hat{h}^{2}\right) \simeq\left(\sigma_{M}^{2}+\sigma_{R}^{2}\right)^{2} V(\hat{E})+E^{2} V\left(\hat{\sigma}_{M}^{2}+\hat{\sigma}_{R}^{2}\right) \\
-2 E\left(\sigma_{M}^{2}+\sigma_{R}^{2}\right) \operatorname{Cov}\left[\hat{E},\left(\hat{\sigma}_{M}^{2}+\hat{\sigma}_{R}^{2}\right)\right]
\end{array}\right\}
$$

La procédure utilisée pour aboutir à $\mathrm{E}$ donne également $\mathrm{V}(\hat{E})$. Il reste donc à calculer les deux derniers termes de (III 1). 
Des équations (I 2) on déduit :

$$
\hat{\sigma}_{M}^{2}+\hat{\sigma}_{R}^{2}=\left[\widehat{C M}_{M}+(n-1) \widehat{C M}_{R}\right] / n
$$

et :

$$
V\left(\hat{\sigma}_{M}^{2}+\hat{\sigma}_{R}^{2}\right)=\frac{2}{p^{2}}\left[\frac{C_{M}^{2}}{m-1}+\frac{(n-1) C_{R}^{2}}{m}\right]
$$

Comme d'autre part $\hat{D}$ est indépendant de $\hat{\sigma}_{M}^{2}$ et de $\hat{\sigma}_{\mathrm{R}}^{2}$ et que, d'après (I 2) $\operatorname{Cov}\left(\hat{G}, \hat{\sigma}_{\mathrm{R}}^{2}\right)=0$ et $\operatorname{Cov}\left(\hat{\mathrm{G}}, \hat{\sigma}_{\mathrm{M}}^{2}\right)=\left(-1 / \mathrm{mn}^{2}\right) \mathrm{V}\left(\widehat{\mathrm{CM}}_{\mathrm{M}}\right)$, il vient :

$\operatorname{Cov}\left[\hat{\mathrm{E}},\left(\hat{\sigma}_{\mathrm{M}}^{2}+\hat{\sigma}_{\mathrm{R}}^{2}\right)\right]=\alpha_{2}\left[\operatorname{Cov}\left(\hat{\mathrm{G}}, \hat{\sigma}_{\mathrm{M}}^{2}\right)+\operatorname{Cov}\left(\hat{\mathrm{G}}, \hat{\sigma}_{\mathrm{R}}^{2}\right)\right]=\left[-2 \alpha_{2} / \mathrm{mn}^{2} \mathrm{p}(\mathrm{m}-1)\right] \mathrm{CM}_{\mathrm{M}}^{2}$

L'expression (III 1) devient alors :

$$
\begin{aligned}
\sigma^{8} V\left(\hat{h}^{2}\right)=\left(\sigma_{M}\right. & \left.+\sigma_{R}\right)^{2} V(\hat{E})+2\left(E^{2} / p n^{2}\right) \\
& +4 \alpha_{2} E\left(\sigma_{M}^{2}+\sigma_{R}^{2}\right) C M_{M}^{2} / m^{2} p(m-1) .
\end{aligned}
$$

Dans le cas d'un dispositif déséquilibré, les équations (I 2) font intervenir les coefficients $k_{I}, k_{2}, k_{3}$ et les degrés de liberté $d_{P}, d_{M}$ et $d_{R}$ définis dans l'annexe $I$. Les deux derniers termes de (III 1) se calculent en fonction de ces coefficients et, en faisant l'approximation des carrés moyens indépendants, on obtient :

$$
\mathrm{V}\left(\hat{\sigma}_{M}^{2}+\hat{\sigma}_{R}^{2}\right)=\left(2 / k_{3}^{2}\right)\left[C M_{M}^{2} / d_{M}+\left(k_{3}-1\right)^{2} C_{M}^{2} / d_{R}\right]
$$

et :

$\operatorname{Cov}\left[\hat{E},\left(\hat{\sigma}_{M}^{2}+\hat{\sigma}_{R}^{2}\right)\right]=\left(-2 \alpha_{2} / k_{1} k_{3}\right)\left[\left(k_{2} / k_{3}\right)\left(C M_{M}^{2} / d_{M}\right)+\left(k_{3}-1\right)\left(1-k_{2} / k_{3}\right) C M_{R}^{2} / d_{R}\right]$ 


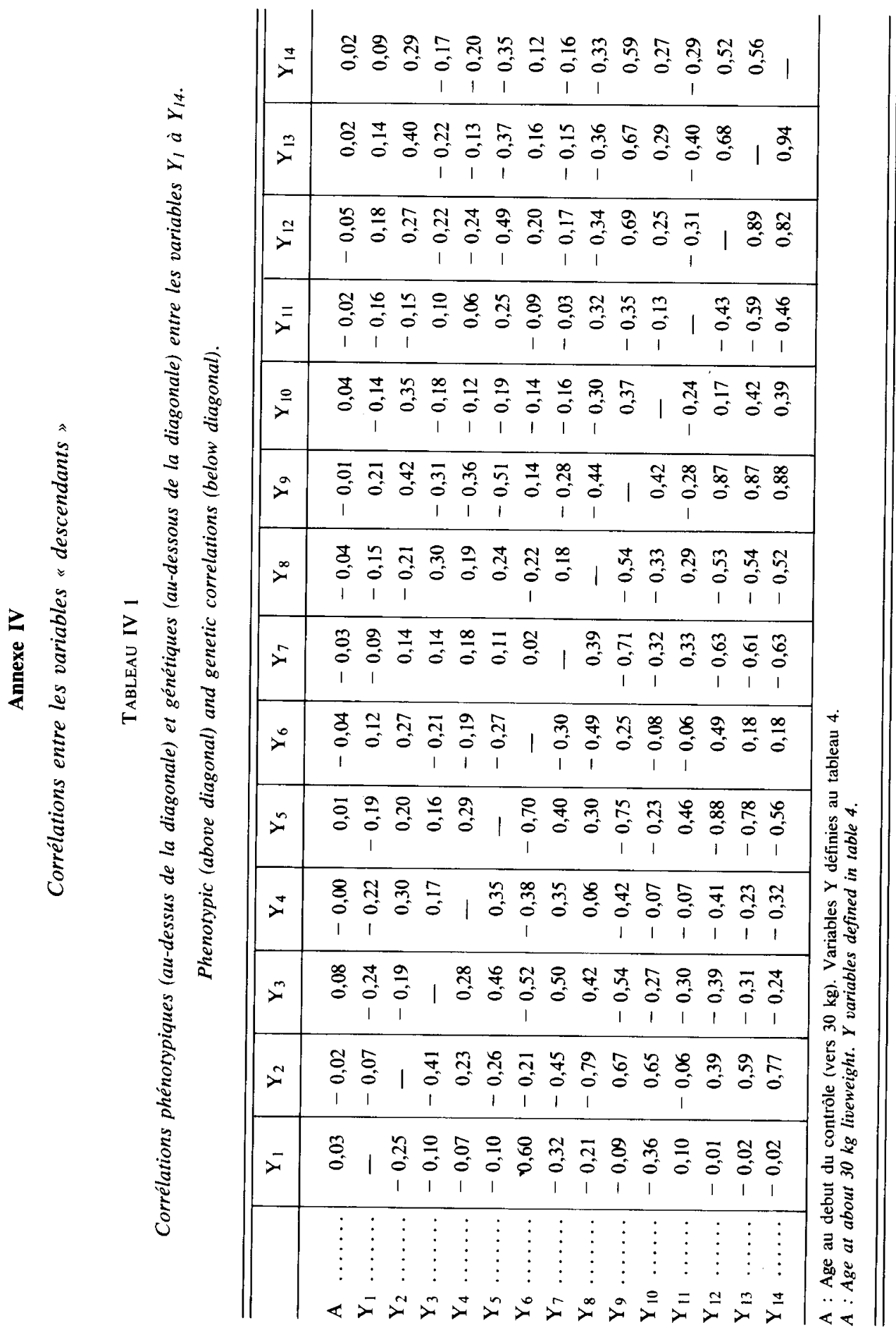




\section{TABLEAU IV 2}

Corrélations phénotypiques (au-dessus de la diagonale) et génétiques (au-dessous de la diagonale) entre les variables "qualité de viande".

Phenotypic (above diagonal) and genetic (below diagonal) correlations between meat quality traits.

\begin{tabular}{|c|c|c|c|}
\hline & $\mathrm{pH}$ & Réflectance & Temps d'imbibition \\
\hline $\mathrm{pH}\left(\mathrm{Y}_{14} \ldots \mathrm{Y}_{18}\right) \ldots$ & $\left(\begin{array}{l}0,62 \\
0,65\end{array}\right)$ & $-0,41$ & 0,34 \\
\hline Réflectance $\left(\mathrm{Y}_{19}, \mathrm{Y}_{20}\right)$ & $-0,53$ & $\left(\begin{array}{l}0,59 \\
0,75\end{array}\right)$ & $-0,32$ \\
\hline Temps d'imbibition $\left(\mathrm{Y}_{21}, \mathrm{Y}_{22}\right)$ & 0,82 & $-0,59$ & $\left(\begin{array}{l}0,59 \\
1,22\end{array}\right)$ \\
\hline
\end{tabular}

Ce tableau indique les moyennes des corrélations obtenues pour les $4 \mathrm{pH}$, les 2 réflectances et les 2 temps d'imbibition (définis au tableau 4). Dans la diagonale figurent les corrélations phénotypiques ( $1^{\text {re }}$ ligne) et génétiques ( $2^{\mathrm{e}}$ ligne) entre les mesures sur muscles différents.

Average correlations are given for $\mathrm{pH}$ measured on 4 muscles, reflectance on 2 muscles and time to get wet on 2 muscles (measurements defined in table 4). The figures in the diagonal are phenotypic (upper line) and genetic correlations (lower line) between measurements on different muscles. 


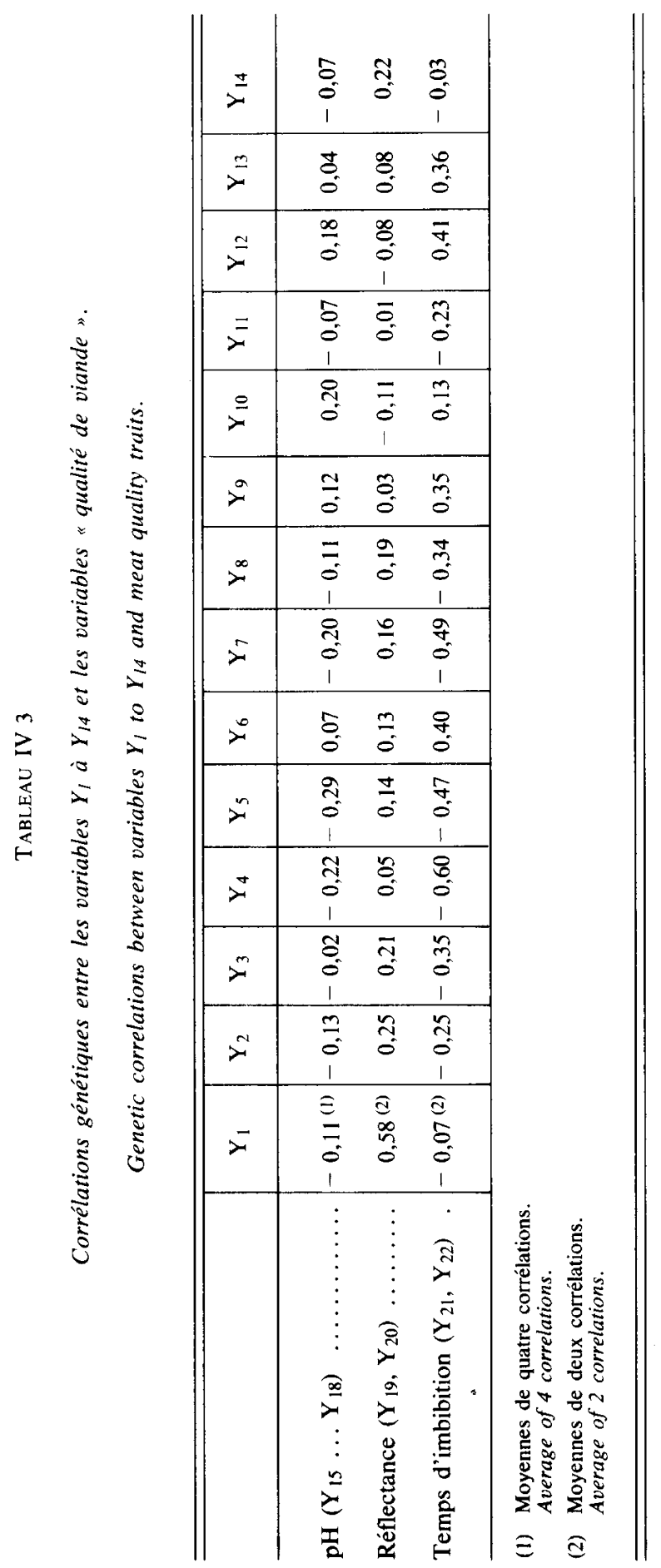

